\title{
Contact dermatitis - Pathomechanism and understanding of disease in clinical setting
}

M. Mathur ${ }^{1}$ S. Kumari ${ }^{2}$

${ }^{1}$ Professor ${ }^{2}$ Senior Resident, Department of Dermatology, College of Medical Sciences, Bharatpur, Chitwan, Nepal

\begin{abstract}
According to the pathophysiological mechanisms involved, two major types of contact dermatitis recognized irritant contact dermatitis (ICD) and allergic contact dermatitis (ACD). The diagnosis of either ICD or ACD is mainly depending on comprehensive clinical data (history and physical examination) as well as by performing appropriate diagnostic patch testing. Two forms of contact dermatitis may differ in early phase of development but development of clinical dermatitis have similar mechanism confusing the clinician and differentiating between ICD and ACD is often difficult in the clinical setting.
\end{abstract}

This review will allow practicing physician to adopt rational clinical approach and the implementation of scientific information for therapeutic as well as preventive strategies.

Key words: Contact dermatitis, irritant contact dermatitis (ICD) and allergic contact dermatitis (ACD).

\section{Introduction}

The skin is routinely exposed and receives assault from exogenous environment agents, chemicals and/or physical agents. Fortunately, most of these exposures do not result in clinically apparent disease. However, in some circumstances, an exposure to these agents results in a cascade of pathogenic events leading to inflammation and clinical contact dermatitis (CD).

$\mathrm{CD}$ is a common dermatosis and according to general population studies, contact dermatitis

Correspondence: M. Mathur

E-mail: maheshmathur2@hotmail.com accounts for $4-7 \%$ of all dermatological consultations. ${ }^{1}$ Skin disease, chiefly dermatitis accounts for almost half of occupational disease and have profound socioeconomic impact. ${ }^{2}$ Over $20 \%$ of females will suffer from hand eczema at some stage in their lives. ${ }^{3}$ Disease represents considerable predicament, in view of the high frequency of occurrence, physical handicap, specially among patients having chronic recalcitrant disease and profound impact on the patient's quality of life. According to the pathophysiological mechanisms involved, two major subtypes of CD are recognized: irritant $\mathrm{CD}$ (ICD) and allergic $\mathrm{CD}$ (ACD). The two types may, and often do, coexist, 
which complicate the matters in clinical setting. Making a correct diagnosis and identifying the causative agent(s) is of utmost importance for the appropriate therapeutic and preventive measures.

\section{Pathomechanism of CD}

Irritant $\mathrm{CD}$ is the clinical result of direct inflammation arising from the release of proinflammatory cytokines from skin cells (principally keratinocytes) in response to chemical or physical harmful stimuli. There are three main pathophysiological changes of irritant dermatitis reported: skin barrier disruption, cellular epidermal change and inflammatory mediator release and all are interconnected. ${ }^{4}$ Disruption of the epidermal barrier function results in the release of proinflammatory cytokines from damaged and activated viable cornified cells. ${ }^{5,6}$ Skin irritants are able to activate the skin's innate immunity independently of the antigen presentation pathway and the induction of proinflammatory mediators that directly recruit and activate $\mathrm{T}$ lymphocytes, without the induction of antigen-specific memory $\mathrm{T}$ cells. Most of the cytokines/factors and cell adhesion molecules previously associated with ACD, such as ICAM-1, (Intercellular Adhesion Molecule-1) lymphocyte function-associated antigen (LFA)-1, IL-1á, IL-1â, TNF-á, granulocyte macrophage-colony-stimulating factor (GM-CSF) and IFN-ã, have been found in the epidermis and dermis in irritant reactions. ${ }^{5}$

ACD is the result of delayed contact hypersensitivity (CHS) reaction elicited by allergens and the inflammatory response is by clonally expanded allergen-primed memory $\mathrm{T}$ lymphocytes. The current paradigm of delayed contact sensitivity follows a two-step mechanism - sensitization and an elicitation phase. In the sensitization phase, low-molecular-weight and the small size of the chemicals allows penetration through a skin barrier that is otherwise impermeable to large molecules under physiological conditions. Once in contact with the skin, antigen (haptens) will activate the skin's innate immunity and induce an inflammatory response, recruiting and activating leukocytes and dendritic cells (DCs). The haptens or conjugated hapten-peptide complexes are internalized through pinocytosis or receptormediated endocytosis and processed by DCs, which up regulate the expression of surface molecules such as MHC molecules and costimulatory factors including IL-1 and TNF-á. Activated DCs migrate to the draining lymph nodes, where they present the haptenated peptides together with the autologus MHC class I and II molecules to specific MHC class I-restricted CD8+ and MHC class II-restricted CD4+ T cells. These cells have effector and regulatory functions on the delayed hypersensitivity reaction and the development of a skin inflammatory reaction against specific allergens will probably depend on the balance between the effects of effector and regulatory/suppressor T cells. There is new evidence that innate immune lymphocytes, such as natural killer $\mathrm{T}$ cells and possibly these cells, may also play an important role in inflammation. ${ }^{7}$

Recent studies have demonstrated that allergen sensitization induces the development of distinct CD8+ T-cell subpopulations that produce IFN-ã (Tc1) or IL-17 (TIL-17). It is reported that both IFNã and IL-17 are required for optimal CHS 
M. Mathur et al, Contact dermatitis - Pathomechanism and understanding........

responses. ${ }^{8}$ CD4 + cells, by contrast, produce the Th2 cytokines IL-4 and IL-10. ${ }^{9}$ T-cell populations primed by hapten sensitization in contact sensitivity are distinguished by polarized patterns of cytokine production: IFN-ã-producing (Tc1) effector CD8+ T cells and IL-4/IL-10-producing (Th2) regulatory CD4+ T cells. Allergen-specific, clonally expanded $\mathrm{T}$ cells leave the lymph nodes and circulate in the blood, secondary lymphatic organs and tissues, including the skin. Lymphocytes expressing skinhoming receptor, such as cutaneous lymphocyteassociated antigen or CCR4, preferentially recirculation into the skin. In the elicitation phase, following subsequent contact with the same chemical or related chemicals, allergen-specific $\mathrm{T}$ cells already present on the skin, will produce an inflammatory response causing the clinical dermatitis. ${ }^{10}$

Hence, the mechanisms at the origin of the clinical lesions of $\mathrm{CD}$ are dissimilar in the two types of dermatitis specially the early stages of the inflammatory response. ICD follows the activation of innate immunity, while in ACD both the innate and acquired immunity are activated and, as a result, antigen-specific effector $\mathrm{T}$ cells will drive the inflammatory response. The later stages of the immune inflammation giving rise to an eczematous lesion may be very similar in ACD and ICD thus in practice, irritancy and allergy are almost always associated and closely linked. Both types of dermatitis share the same effector pathways and involve the same cytokines, chemokines, apoptosis phenomena and cellular necrosis, as well as the recruitment of a polymorphic inflammatory infiltrate. ${ }^{11}$ In addition, most strong allergens also have irritant properties. ${ }^{12}$ and irritancy is believed to play a crucial role in the development of ACD. Skin cell damage and cytokine release induced by irritants may represent a 'danger signal' for the immune system, activating antigen-presenting DCs and recruiting DC precursors, which are blood monocytes, into the skin, thus predisposing to contact sensitization. ${ }^{13}$ In the absence of activation of innate immunity, the maturation of skin DCs is incomplete and there is no appropriate activation of pro-inflammatory effector $\mathrm{T}$ cells. Conversely, immature DCs are capable of activating antiinflammatory regulatory $\mathrm{T}$ cells. This knowledge can be applied directly to clinical practice and the early recognition and treatment of ICD will contribute to preventing the development of ACD. The genetic basis for increased susceptibility to $C D$ is being intensely studied. A large proportion of individuals with Atopic Dermatitis, (16-56\%) have an epidermal expression deficiency of filaggrin (FLG) - important for barrier function of skin and individuals with atopic dermatitis carried one or more Filaggrin (FLG) null alleless ${ }^{14}$. The induced alterations in the skin barrier function may predispose to $\mathrm{CD}$ by allowing a greater penetration of chemical irritants or haptens. It is observed that FLG null alleles were associated with increased susceptibility to chronic ICD but not an independent risk factor. ${ }^{15}$ Moreover; population-based study did not find the association. ${ }^{16}$ However, an association was found between FLG null alleles and a positive patch testing for nickel (Ni) but not with contact sensitizations to other allergens. ${ }^{17} \mathrm{Ni}$ is chelated in the epidermis and perhaps to FLG. and may be 
responsible for association. Therefore, more research is needed to determine the causal relationship.

\section{Clinical Diagnosis of CD}

The diagnosis of CD depends on patient history, clinical examination, exposure assessment, risk characterization, analysis of all predisposing and contributory factors, and comprehensive diagnostic testing. Although a preliminary working diagnosis of CD may often be made after a thorough clinical assessment but to decide whether the dermatitis is primarily irritant or allergy is not always straightforward and often misleading. There are no pathognomonic clinical signs and symptoms which can discriminate between ACD and ICD.

The first step in the diagnosis of $\mathrm{CD}$ is a comprehensive history that covers the clinical evolution of the dermatitis and all possible etiological factors. Assessment of exposure should investigate including working activities and occupational environment. The nature of the current and previous jobs involving exposure to irritant or allergenic products. Material safety data sheets may identify materials and their side effects. Nonoccupational sources, including hobbies and leisure activities, and domestic exposures, such as personal skin care products and fragrances, clothing and accessories, plants, and previous and current treatments should be recorded. In addition, potential cross-sensitizers that may have come in contact in past should be considered. Record of simultaneous exposure to several allergens and/or irritants and exposure factors, such as occlusion, friction, trauma and heat, which might enhance the percutaneous penetration by damaging the skin barrier, is useful
In most cases of $\mathrm{CD}$, several exposures are required for sensitization or irritation to develop but contact sensitization or irritation may result from a single exposure to a strong allergen or irritant. In case of cumulative ICD, multiple sub-threshold skin insults due to multiple weak irritants may results in clinical dermatitis. Sometimes, the source of exposure remains concealed because patients are often unable to recognize a causal relationship specially when exposure is infrequent or sporadic. We should be aware that there are different possibilities of exposure including: contact with allergencontaminated items; transfer of an allergen with the hands to the face, neck or other sites, which produces 'ectopic' $\mathrm{CD}$; exposure to a product that is used by partner or another person who is close to the patient, may cause dermatitis - which is called 'connubial' or 'consort' dermatitis, or dermatitis 'by proxy'. Transfer of the allergen through the air - gasses or vapours, droplets, or powders - which may give rise to an 'airborne' dermatitis. Systemic exposure to the allergen (or to a cross-reacting substance) after previous sensitization of the skin, may produce flare-up of lesions at the previous contact sites or a generalized eczematous reaction may develop as 'systemic CDtype reaction'. Finally, there is possibility of photoallergic or phototoxic $\mathrm{CD}$ (the consequence of exposure to a photo allergen or a phototoxic substance and sunlight).

Clinical Symptoms of ICD may include burning, itching, stinging, soreness and pain, particularly at the beginning of the clinical course but pruritus is the cardinal symptom in ACD. It is mandatory to determine time relationship between the attributable exposure and the clinical course of the 
M. Mathur et al, Contact dermatitis - Pathomechanism and understanding......

dermatitis. The patient's description of events may be important, including the date of onset of the dermatitis, its clinical characteristics and initial affected sites. The rapidity of onset after exposure and the clinical course is helpful in distinguishing ICD with ACD.

In $\mathrm{ACD}$, two phases are required: sensitization followed by elicitation of a cutaneous inflammatory reaction. Except for very potent allergens, the primary sensitization does not result in clinical skin lesions, probably due to the low numbers of responder $\mathrm{T}$ lymphocytes in this phase and upon subsequent challenge results in the clinical lesions. However, in contrast low-grade irritants will induce dermatitis only after multiple exposures. Moreover, many allergens have cross-reactants that can give a reaction on first exposure, without a period of induction. Concerning the time course for the onset of clinical CD, newly exposed workers with occupational dermatosis demonstrated no significant time differences in the development of ACD and ICD. ${ }^{18}$

The acute irritant reaction usually reaches its peak quickly, within minutes to a few hours after exposure, and then starts to heal. Certain irritants may elicit a delayed inflammatory response, and visible inflammation is not seen until 12-24 hours or even longer after exposure. Sodium lauryl sulphate, the most studied irritant substance, may give a more intense inflammatory reaction at $48 \mathrm{~h}$ after exposure, thus time course is more characteristic of allergic reactions. ${ }^{19}$ In ACD, the elicitation time depends on the characteristics of the sensitizer, the intensity of exposure and degree of sensitivity. Lesions usually appear 24-72 hours after the exposure to the causative agent and reach their peak at approximately 72-96 hours. However, they may develop as early as $5 \mathrm{~h}$ or as late as 7 days after exposure. Often, ACD improves more slowly than ICD when exposure ceases, and recurs faster (in a few days) when exposure is reestablished. A clinical course characterized by sudden flares of dermatitis frequently indicates ACD. A feature of ACD that may be confused is the sudden development of a delayed hypersensitivity after years of contact with a substance. While it is true that many cases of ACD develop following recent (weeks to months) exposure to an allergen, ACD may also develop after years of repeated exposure.

A systematic consideration of the clinical features is needed for correct diagnosis of CD and mostly characterized by eczematous skin lesions. However, rarely, ACD may adopt other clinical patterns, such as erythema multiforme-like ${ }^{20}$ urticarial papular plaques, lichen-planus-like and lichenoid eruptions, ${ }^{21}$ purpuric petechial reactions ${ }^{22}$, dermal reactions ${ }^{23}$, lymphomatoid $\mathrm{CD}^{24}$, granulomatous and pustular reactions ${ }^{25}$, pigmentation disturbances and even pemphigoid lesions. ${ }^{26}$ have been described.

In most cases, the shape and pattern of distribution of the lesions primarily confined to the site of contact and the distribution of lesions is the most important clue for the diagnosis of CD but atypical presentation may be misleading sign. It is possible for CD to occur unilaterally, even though exposure is bilateral, or to occur at sites distant form the actual exposure due to transfer of the agent with the hands or fomites. The body sites, where the stratum corneum is thinner, thereby allowing greater penetration of the contactant - as in the case 
of eyelids - the rash may be more severe than at other contact points. However, in the late stage of disease, the original pattern of the dermatitis is frequently modified by secondary dissemination, infection or treatment. Therefore, early diagnosis is of the utmost importance.

Irritant reactions believed to be nonspecific and reproducible in all exposed subjects, in contradistinction with the allergic reactions. The effects of irritants on skin are highly variable, depending on several factors, such as the type of agent, concentration, amount applied to the skin surface, surface area, regional variations, length of exposure, mode of exposure, concomitant environmental factors and individual susceptibility, including age, sex, race, genetic background and concomitant disease. ${ }^{27,28,29}$ Irritant thresholds and dose responses vary considerably among individuals and also in the same individual over time. ${ }^{27}$ ICD lesions are usually sharply demarcated and confined to the contact area, while in ACD; lesions are less circumscribed and frequently disseminated. However, ICD lesions may disseminate depending on the characteristics of the exposure. Irritant reactions due to detergents often involve the finger webs, concentrate under rings, and may extend over the dorsum of the hands in an apron-like distribution. Pustules, necrosis and ulceration may be seen after contact with strong irritants, whereas they are rarely observed in ACD. Vesiculation, edema and weeping are usually more prominent in $\mathrm{ACD}$, but certain irritants may induce a polymorphous dermatitis with vesiculation mimicking ACD. Furthermore, ACD in certain body areas, such as the eyelids, penis and scrotum, may exhibit only erythema and edema without vesiculation. The diagnosis of acute ICD to strong skin irritants is usually straightforward, because the rapid onset of skin lesions after exposure indicates the causative agent. On the other hand, sub acute or chronic ICD or ACD frequently appear as an eczematous condition with erythema, excoriations, lichenification, scaling and/or hyperkeratosis, and the causative agents are not always apparent. The distinction may be even more complicated because many allergens have irritant effects and/or both types of contact agents act jointly

\section{Conclusion}

Contact Dermatitis is a common dermatosis among patients visiting to dermatology out patients worldwide. Disease is having significant impact on the quality of life of the affected patients and profound socieconomical stigma. However, two types of CD may coexist and it is difficult to discern between ICD and or ACD in clinical settings It is impossible to discriminate between ICD, ACD on clinical grounds, as erythema, edema, scaling and vesiculation in acute dermatitis, and fissuring, lichenification and hyperkeratosis in the chronic phase, are largely nonspecific signs. Patch test is confirmative of contact sensitization current and/ or past and remains only useful and reliable method for the diagnosis of AC therefore, details of history and systematic clinical examination are of paramount importance for the diagnosis of $\mathrm{CD}$ and often rewarding. ${ }^{30}$ 
M. Mathur et al, Contact dermatitis - Pathomechanism and understanding.

\section{References}

1. C.G.T. Mathias. Contact dermatitis and workers' compensation: criteria for establishing occupational causation and aggravation. J. Am. Acad. Dermatol. 1989;20 (5):842.

2 B.J. Nickoloff, Y. Naidu. Perturbation of epidermal barrier function correlates with initiation of cytokine cascade in human skin. J. Am. Acad. Dermatol . 1994;30(4):535-6.

3 R.C. McKenzie, D.N. Sauder. The role of keratinocyte cytokines in inflammation and immunity. J. Invest. Dermatol. 1990; 95 (6 Suppl.),105S -7.

4. M.D. Gober, R. Fishelevich, Y. Zhao, et al. Human natural killer T cells infiltrate into the skin at elicitation sites of allergic contact dermatitis. J. Invest. Dermatol. 2008;128(6):1460 -9.

5. D. He, L. Wu , H. K. Kim, et al. IL-17 and IFN-ã mediate the elicitation of contact hypersensitivity responses by different mechanisms and both are required for optimal responses. J. Immunol. 2009; 183 (2):1463 - 70.

6. H Xu , A Banerjee, NA Dilulio, ert all. Development of effector CD8+ T cells in contact hypersensitivity occurs independently of CD4+ T cells. J. Immunol 1997;158 (10), $4721-28$.

7. O. Baadsgaard, T. Wang . Immune regulation in allergic and irritant skin reactions. Int. J. Dermatol 1991; 30(3):161-72 .

8. T. Hunziker, C.U. Brand, A. Kapp, et al,. Increased levels of inflammatory cytokines in human skin lymph derived from sodium lauryl sulphate-induced contact dermatitis. Br. J. Dermatol. 1992;127(3):254-7

9. A.K. Ulfgren, L. Klareskog, M. Lindberg . An immunohistochemical analysis of cytokine expression in allergic and irritant contact dermatitis. Acta Derm. Venereol. 2000;80(3):167-70 .

10. J. Brasch, J. Bugard, W. Sterry. Common pathogenetic pathways in allergic and irritant contact dermatitis. $J$. Invest. Dermatol. 1992; 98 (2):166-70.
11. D.J. Gawkrodger, E. McVittie, M.M. Carr, et al. Phenotypic characterization of the early cellular responses in allergic and irritant contact dermatitis. Clin. Exp. Immunol. 1986;66(3):590-8.

12 L.K. Pedersen, J.D. Johansen, E. Held, et al. Augmentation of skin response by exposure to a combination of allergens and irritants - a review. Contact Derm. 2004;50(5):265-73.

13. J.P. McFadden, D.A. Basketter. Contact allergy, irritancy and 'danger'. Contact Derm 2000;42(3),123-7.

14. H. Baurecht, A.D. Irvine, N. Novak et al. Toward a major risk factor for atopic eczema: meta-analysis of filaggrin polymorphism data. J. Allergy Clin. Immunol. 2007;120(6):1406-12 .

15. C.M. de Jongh , L. Khrenova, M.M. Verberk et al. Lossof-function polymorphisms in the filaggrin gene are associated with an increased susceptibility to chronic irritant contact dermatitis: a case-control study. $B r . J$. Dermatol.2008;159(3):621-7.

16 A. Lerbaek, H. Bisgaard, T. Agner, et al. Filaggrin null alleles are not associated with hand eczema or contact allergy. Br. J. Dermatol. 2007;157(6):1199-1204.

17. N. Novak, H. Baurecht, T. Schäfer et al. Loss-of-function mutations in the filaggrin gene and allergic contact sensitization to nickel. J. Invest. Dermatol. 2008; 128(6):1430-5.

18. S. Fregert, Occupational dermatitis in a 10-year material. Contact Derm.1975; 1(2):96-107.

19. D.P. Bruynzeel, W.G. van Ketel, R.J. Scheper et al. Delayed time course of irritation by sodium lauryl sulfate: observations on threshold reactions. Contact Derm. 1982;8(4):236-9.

20. S. Kerre, A. Busschots, A. Dooms-Goossens. Erythemamultiforme-like contact dermatitis due to phenylbutazone. Contact Derm. 1995; 33(3):213-14. 
Journal of College of Medical Sciences-Nepal, 2011, Vol-7, No-4

21. J.A. Yiannias, R.A. el-Azhary, J.H. Hand et al. Relevant contact sensitivities in patients with the diagnosis of oral lichen planus. J. Am. Acad. Dermatol. 2000;42(2 Pt 1):177-82.

22. J. Roed-Petersen, O.J. Clemmensen, T. Menné et al . Purpuric contact dermatitis from black rubber chemicals. Contact Derm. 1988;18(3):166-8.

23 N.Katoh, S. Hirano, S. Kishimoto, et al. Dermal contact dermatitis caused by allergy to palladium. Contact Derm. 1999; 40(4):226-7.

24. J.G.Orbaneja, L.I. Diez, J.L.S. Lozano, et al. Lymphomatoid contact dermatitis: a syndrome produced by epicutaneous hypersensitivity. Contact Derm. 1976;2(3):139-43.

25 J.M.Sánchez-Motilla,V. Pont, E. Nagore et al. Pustular allergic contact dermatitis from minoxidil. Contact Derm. 1998;38(5):283-4.
26. L Stransky, The so-called contact dermatoses. Contact Derm. 1998;38(4):216-7.

27. M.R. Judge, H.A. Griffiths, D.A. Basketter. Variation in response of human skin to irritant challenge. Contact Derm. 1996; 34(2):115-7.

28 K. Lammintausta, H.I. Maibach, D. Wilson, Irritant reactivity in males and females. Contact Derm. 1987; 17(5):276-80.

29. S. Patil, H.I. Maibach, Effect of age and sex on the elicitation of irritant contact dermatitis. Contact Derm. 1994;30(5):257-64.

30. G. Agrup, I. Dahlquist, S. Fregert et al. Value of history and testing in suspected contact dermatitis. Arch. Dermatol. 1970;101:212-5. 